\title{
Improving smoking cessation referrals among elective surgery clinics through electronic clinical decision support
}

\author{
Connor Stonesifer ${ }^{1}$, Salvatore Crusco ${ }^{2,3}$, Sritha Rajupet ${ }^{1,2,3}$
}

\begin{abstract}
INTRODUCTION Preoperative visits are an exceptional opportunity to encourage smoking cessation, as studies demonstrate the experience of scheduling elective surgery produces an actionable incentive to quit. However, studies suggest surgeons do not regularly assess smoking behavior or offer cessation therapies. Clinical decision support (CDS) is a system in which providers are presented with clinically integrated tools to enhance decision-making.

METHODS A CDS tool was designed to facilitate treatment referrals for smoking cessation services among patients seeking elective surgery. Two clinics were selected: the plastic and vascular surgeries. The study objectives were to assess the utilization rate and effectiveness of this system.

RESULTS No smoking cessation referrals had been submitted by the plastic surgery or vascular surgery clinics in the year before CDS tool implementation. Providers at the plastic surgery clinic utilized the CDS tool in 95.0\% (191 of 201) eligible patient encounters. Of these patients, $16.3 \%$ were identified as active smokers, and $16.1 \%$ of these smokers accepted treatment referrals. Providers at the vascular surgery clinic utilized the CDS tool in 50.3\% (98 of 195) eligible patient encounters. Of these patients, $10.2 \%$ were identified as active smokers, and $30.0 \%$ of these smokers accepted treatment referrals.

CONCLUSIONS The CDS tool improved the incidence of smoking cessation referrals in two surgical clinics from pretest baselines and achieved satisfactory utilization rates. This report demonstrates the feasibility of CDS tools to actualize the preoperative visit as an opportunity to promote smoking cessation.
\end{abstract}

\section{AFFILIATION \\ 1 Vagelos College of Physicians and Surgeons, Columbia University, New York, United States \\ 2 Icahn School of Medicine at Mount Sinai, New York, United States \\ 3 James J. Peters VA Medical Center, Bronx, United States}

\section{CORRESPONDENCE TO \\ Connor Stonesifer. Vagelos College of Physicians and Surgeons, Columbia University, New York, NY 10032, United States. E-mail: cjs2244@cumc.columbia.edu ORCID ID: https://orcid.org/0000- 0003-1513-336X}

\section{KEYWORDS}

surgery, feasibility study, clinical decision support, smokingcessation, tobacco-related disparities, tobacco-use cessation

Received: 4 September 2020 Revised: 28 October 2020

Accepted: 19 December 2020

\section{INTRODUCTION}

According to the World Health Organization, the United States ranks second in global surgical volume, with over 36 million surgeries performed each year as of $2012^{1}$. The prevalence of tobacco use among the US surgical population is disproportionately high, with estimates suggesting that $30 \%$ of patients undergoing elective general surgery are active smokers ${ }^{2}$. Preoperative smoking is an independent risk factor for significant postoperative morbidity and mortality ${ }^{3-5}$.

Recent analyses suggest smokers are up to 1.53 times more likely to die in the 30 days after surgery than non-smokers ${ }^{6}$. Preoperative smokers also experience higher rates of postoperative pneumonia, post-surgical intubation, myocardial infarction, stroke, sepsis, and wound complications than both non-smokers and former smokers ${ }^{3-5,7}$. The added burden posed by smoking on both perioperative patients and the health system has been described across surgical disciplines, leading the American College of Surgeons to declare that smoking cessation counseling should be offered during all non-emergent patient consultations ${ }^{6}$.

Among US smokers, 68\% report wanting to quit completely ${ }^{7}$. Despite patient interest and proven postoperative risks, only $58 \%$ of surgeons 'almost always' 
advise their patients who use tobacco to quit. Moreover, only $13 \%$ of surgeons 'almost always' provide resources to help their patients who use tobacco to quit ${ }^{8}$. Surgical clinics pose an exceptional opportunity to encourage behavior change, with studies demonstrating that the experience of scheduling elective surgery produces a significant, actionable incentive to stop smoking ${ }^{2,9}$.

Clinical decision support (CDS) is a system in which physicians are provided with knowledge that is intelligently filtered and presented at appropriate times to enhance healthcare delivery ${ }^{10}$. CDS systems may include computerized alerts and reminders, condition-specific order sets, and contextually relevant reference information ${ }^{10,11}$.

A CDS tool was designed to increase smoking cessation counseling referrals among patients seeking elective surgery at the James J. Peters VA Medical Center (JJP-VAMC) with a secondary objective to assess the utilization rate of this referral system.

\section{METHODS}

\section{Design and setting}

This was a pretest-posttest study of a CDS tool created in the VA electronic medical record, Computerized Patient Record System (CPRS). The study was managed by the Preventive Medicine Department at the JJP-VAMC and attached to both plastic and vascular surgery clinic notes from July 2019 to February 2020. This CDS tool was designed as a low-click, streamlined means of referring patients to available smoking cessation clinics at the facility. The study was classified as a quality improvement project per VA guidelines and was approved for use in all patients seen in plastic and vascular surgery clinics.

\section{Intervention}

An electronic CDS tool was implemented to assess smoking status and desire for treatment at the initiation of plastic and vascular surgery clinic notes. It was designed as a series of three conditional statements to facilitate ease of use. 'Treatment' referred to the multiple treatment modalities offered at JJP-VAMC, including either a smoking cessation clinic appointment, where counseling as well as pharmacologic therapies are offered, or weekly support groups. The tool included the following questions: 1) 'Has the patient used tobacco products within the last 30 days?', 2) If 'yes', 'Are you interested in smoking cessation treatment?' and 3) If 'yes', the clinician would choose 'individual treatment' or 'group therapy' (Figure 1). By clicking 'individual treatment', the prompt would generate an automatic, pre-populated referral order, with the provider responsible only for providing a suggested consultation date. If 'Quit Smoking Walk-In Group' was selected, the tool displayed a printable flyer. If 'no' or 'cancel' was selected at any time, the template would close with no further actions required. A 'cancel' button was found at the bottom of each screen of the tool and could be selected at any time. The tool was designed to record all inputs and premature cancelations.

\section{Samples}

The pretest sample group was defined as the number of referrals to smoking cessation services made by plastic surgery and vascular surgery clinics from June 2018 to June 2019. The posttest sample group was defined as the number of referrals to smoking cessation services using the CDS tool throughout the implementation period. These de-identified data were accessed via the JJP-VAMC Department of Medical Informatics.

\section{Implementation}

The first phase of implementation ran from July to November 2019 when the tool was implemented in the plastic surgery clinic only. This clinic had lower volume and therefore served as a pilot environment to assess barriers to CDS deployment before expanding to the larger vascular surgery clinic. The second phase ran from November 2019 to February 2020. This

Figure 1. CDS tool (first screen). This image is the first screen of the CDS tool that appeared when a consultation note was created. The tool was designed to minimize interactivity (mouse clicks), provide only the essential information needed to refer a patient to smoking cessation services, and limit time spent in the tool (reading/clicking) by the clinical user. The following screen (not displayed) allowed placement of a pre-populated referral order

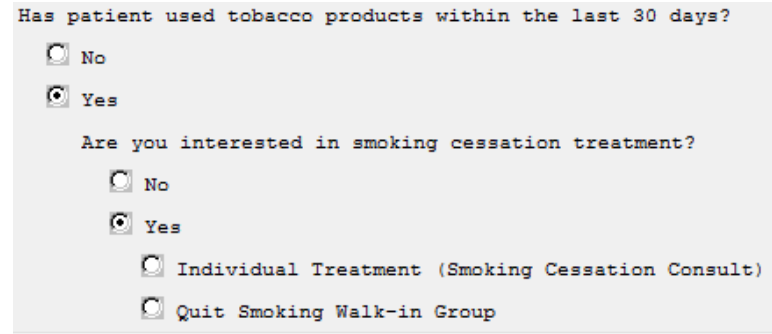


phase saw an expansion of the tool to notes in vascular surgery clinics with continued use by plastic surgery.

\section{Measures and analyses}

CDS tool effectiveness was assessed via comparison of referral rates between pretest and posttest samples. Utilization rate was measured as the number of patient encounters for which the tool was used.

\section{RESULTS}

A pretest data request of plastic and vascular surgery clinic consultations revealed that zero patients were referred to smoking cessation clinics over the 12 months before study implementation. This encompassed 278 and 564 eligible consultations, respectively. Posttest examination demonstrated that for the plastic surgery clinic, between July 2019 and February 2020, a total of 201 new patient consultations were completed. Providers utilized the CDS tool for 191 (95.0\%) patients, with 10 patients (5.0\%) bypassed by providers clicking cancel on the collection instrument. Of the 191 patients, $160(83.7 \%)$ were noted to be non-smokers and $31(16.3 \%)$ were active smokers. Twenty-six of the smokers $(83.9 \%)$ refused treatment, and five smokers (16.1\%) accepted individual treatment with a referral to the smoking cessation clinic.

Within the vascular surgery clinic, a total of 195 new patient consultations were completed between November 2019 and February 2020. For the 195 consultations, providers utilized the CDS tool for 98 (50.3\%) patients, with 97 patients (49.7\%) bypassed by providers clicking cancel on the collection instrument. Of the 98 patients, seventy-five (76.5\%) were noted to be non-smokers, $10(10.2 \%)$ were logged as active smokers, and thirteen (13.3\%) additional patients were lost due to cancelation after initiating data entry. Of the identified smokers, the element was canceled for two $(20 \%)$, five $(50.0 \%)$ refused treatment, and three $(30.0 \%)$ accepted individual treatment with a referral to the smoking cessation clinic. Comparing pretest and posttest values using a one-tailed Z-test, CDS tool implementation produced a significant increase in referrals for both plastic $(p=0.00415)$ and vascular $(p=0.00159)$ clinics.

\section{DISCUSSION}

The purpose of this study was to examine the effectiveness and utilization rate of an electronic CDS tool designed to improve referral rates to smoking cessation services in outpatient surgical clinics. The CDS tool successfully improved the incidence of smoking cessation referrals in two surgical clinics from pretest baselines. These clinics had referred zero patients to smoking cessation services in the year before implementation. In the eight months during which tool use was measured, they collectively referred eight smokers to cessation services. Utilization rates varied between surgical clinics, with frequencies of $95.0 \%$ in the plastic surgery clinic and $50.3 \%$ in the vascular surgery clinic.

\section{Limitations}

This study had limitations. First, CPRS required that the tool automatically open when a new patient consultation note was generated. Surgeons endorsed that they typically assess smoking behavior later in the course of patient interviews. The timing of the CDS tool pop-up was dyssynchronous with the optimal workflow of the clinical visit, and the tool did not trigger again if canceled. Moreover, the CDS tool was not mandatory to acknowledge. Both vascular and plastic surgery clinics are high-volume, time-pressured environments, where disruptions in clinic pacing can be detrimental. These factors likely contributed to cancelation rates; CDS tools not integrated into the clinical workflow and not mandatory to acknowledge are more likely to be overridden ${ }^{12,13}$. Next, data was limited to plastic and vascular surgery clinics, which may impact the generalizability of these results to all outpatient surgical clinics. Additionally, though utilization rates are a reasonable predictor for feasibility, further qualitative data on provider's opinions of tool usability could have informed optimization of the instrument. Finally, multiple attempts to provide education regarding tool use were provided to the vascular clinic, but this was not feasible due to scheduling. Cancelation is presumably the cause behind the low percentage of vascular patients identified as smokers.

Despite receiving no formal education on CDS tool use and the aforementioned limitations, the observed utilization rate among vascular surgery residents and attendings was $50.3 \%$, a value in line with successful utilization reported in CDS literature ${ }^{12-17}$. The plastic surgery clinic achieved a $95.0 \%$ utilization rate following directed education, suggesting there is potential for significant uptake by surgical providers.

The VA health system is a unique environment to 
test CDS tools aimed at facilitating smoking cessation as VA Directive 1056 states that all facilities must provide patients with opportunities for behavioral counseling and pharmaceutical assistance in quitting smoking ${ }^{18}$.

\section{CONCLUSIONS}

This study demonstrates that a simple CDS tool can significantly improve referrals by outpatient surgical providers to smoking cessation services. Our preliminary data strongly suggest that future studies should examine the efficacy of expanding comparable CDS tools among outpatient surgical clinics, as these tools enable the preoperative visit to serve as a more impactful opportunity to encourage smoking cessation.

\section{REFERENCES}

1. Weiser TG, Haynes AB, Molina G, et al. Size and distribution of the global volume of surgery in 2012. Bull World Health Organ. 2016;94(3):201-209. doi:10.2471/blt.15.159293

2. Kamath AS, Sarrazin MV, Vander Weg MW, Cai X, Cullen J, Katz DA. Hospital costs associated with smoking in veterans undergoing general surgery. J Am Coll Surg. 2012;214(6):901908. doi:10.1016/j.jamcollsurg.2012.01.056

3. Khullar D, Maa J. The impact of smoking on surgical outcomes. J Am Coll Surg. 2012;205(3). doi:10.1016/j.jamcollsurg.2012.05.023

4. Grønkjær M, Eliasen M, Skov-Ettrup LS, et al. Preoperative smoking status and postoperative complications: A systematic review and meta-analysis. Ann Surg. 2014;259(1):52-71. doi:10.1097/SLA.0b013e3182911913

5. Turan A, Mascha EJ, Roberman D, et al. Smoking and perioperative outcomes. Anesthesiology. 2011;114(4):837846. doi:10.1097/ALN.0b013e318210f560

6. Alberg AJ, Shopland DR, Cummings KM. The 2014 Surgeon General's Report: Commemorating the 50th Anniversary of the 1964 Report of the Advisory Committee to the US Surgeon General and Updating the Evidence on the Health Consequences of Cigarette Smoking. Am J Epidemiol. 2014;179(4):403-412. doi:10.1093/aje/kwt335

7. Babb S, Malarcher A, Schauer G, Asman K, Jamal A. Quitting smoking among adults - United States, 20002015. Morb Mortal Wkly Rep. 2017;65(52):1457-1464. doi:10.15585/mmwr.mm6552a1

8. Warner DO, Sarr MG, Offord KP, Dale LC. Anesthesiologists, general surgeons, and tobacco interventions in the perioperative period. Anesth Analg. 2004;99(6):17661773. doi:10.1213/01.ANE.0000136773.40216.87

9. Warner DO. Tobacco dependencde in surgical patients. Curr Opin Anaesthesiol. 2007;20(3):279-283. doi:10.1097/ACO.0b013e3280c60c3b

10. Office of the National Coordinator for Health Information Technology. Clinical Decision Support. HealthIT.gov. https:// www.healthit.gov/topic/safety/clinical-decision-support.
Updated April 10, 2018. Accessed December 25, 2019.

11. Bates DW, Kuperman GJ, Wang S, et al. Ten Commandments for Effective Clinical Decision Support: Making the Practice of Evidence-based Medicine a Reality. J Am Med Informatics Assoc. 2003;10(6):523-530. doi:10.1197/jamia.M1370

12. Van de Velde S, Heselmans A, Delvaux N, et al. A systematic review of trials evaluating success factors of interventions with computerised clinical decision support. Implement Sci. 2018;13(1):1-11. doi:10.1186/s13012-018-0790-1

13. Bell H, Garfield S, Khosla S, Patel C, Franklin BD. Mixed methods study of medication-related decision support alerts experienced during electronic prescribing for inpatients at an English hospital. 2019;26(6):318-322. doi:10.1136/ejhpharm-2017-001483

14. Demakis JG, Beauchamp C, Cull WL, et al. Improving residents' compliance with standards of ambulatory care: Results from the VA cooperative study on computerized reminders. J Am Med Assoc. 2000;284(11):1411. doi:10.1001/jama.284.11.1411

15. Murphy E V. Clinical decision support: Effectiveness in improving quality processes and clinical outcomes and factors that may influence success. Yale J Biol Med. 2014;87(2):187-197. PMID:24910564.

16. Jenssen BP, Bryant-Stephens T, Leone FT, Grundmeier RW, Fiks AG. Clinical decision support tool for parental tobacco treatment in primary care. Pediatrics. 2016;137(5):e20154185. doi:10.1542/peds.2015-4185

17. Marcy TW, Kaplan B, Connolly SW, Michel G, Shiffman RN, Flynn BS. Developing a decision support system for tobacco use counseling using primary care physicians. Inform Prim Care. 2008;16(2):101-109. PMID:18713526.

18. Veterans Health Administration. National Smoking and Tobacco Use Cessation Program. VHA Directive 1056. Washington, DC: Department of Veterans Affairs, Veterans Health Administration; 2019.

\section{ACKNOWLEDGEMENTS}

The authors would like to acknowledge Adelina Fuentes-Ramos, Clinical Applications Coordinator at the JJP-VAMC, for her invaluable assistance in implementing the CDS tool, as well as Irvin Rosado, Chief Health Informatics Officer at the JJP-VAMC, for assisting with data collection.

\section{CONFLICTS OF INTEREST}

The authors have completed and submitted the ICMJE Form for Disclosure of Potential Conflicts of Interest and none was reported.

\section{FUNDING}

There was no source of funding for this research.

\section{AUTHORS' CONTRIBUTIONS}

CS was the primary author in drafting the manuscript and devised the project. SC assisted in drafting the manuscript and served as the on-site educator who taught tool use during the implementation period while also collecting provider feedback related to the tool. SR oversaw the project planning and provided edits to the design and manuscript.

PROVENANCE AND PEER REVIEW

Not commissioned; externally peer reviewed. 